\title{
Impact of the 2015 July Heat Waves in France on Heat- Related Causes
}

\author{
Céline Caserio-Schönemann*, Anne Fouillet, Aymeric Ung, Manuel Zurbaran, Vanina \\ Bousquet, Karine Laaidi, Mathilde Pascal, Sébastien Denys, Thierry Cardoso and Anne \\ Gallay
}

French Institute for Public Health Surveillance, Saint Maurice, France

\section{Objective}

To present the evolution of heat-related pathologies during heat waves occurring in July 2015 in France

\section{Introduction}

Two major heat waves occurred in France in July 2015. A first episode characterized by early onset, intensity, large geographical coverage and duration occurred between 29th of June $8^{\text {th }}$ of July. A second episode less intense was localized on the South-East of the country from $15^{\text {th }}$ to $23^{\text {rd }}$ of July.

The French heat warning system has been operating by InVS since 2004 as part of the French National Heat Wave Plan. Warnings are based on meteorological forecasts and on real-time follow-up of specific health indicators to support decision-making. The evolutions in emergency health care facilities during the July heat waves are presented.

\section{Methods}

Real-time morbidity indicators are produced by the French national syndromic surveillance system $\operatorname{SurSaUD}{ }^{\circledR}$ which is based on the daily collection of data from emergency department (ED)

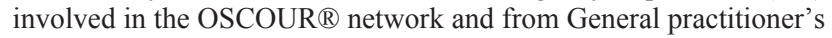
emergency associations SOS Médecins (GPSM). Individual data are automatically recorded and transmitted daily to InVS including administrative, demographic and medical information (coded medical diagnosis) [1].

ED attendances and GPSM visits for all-causes and for heatrelated causes are specifically followed during heat waves. Heatrelated causes include heat stroke/hyperthermia, dehydration and hyponatremia in ED and heat stroke and dehydration in GPSM. These pathologies only represent a small part of the potential health impact during heat waves. They were selected for their reactivity and to provide indications on the spatio-temporal dynamics of the health impacts. Indicators are investigated by age, with a special focus on people aged 75 and over. The proportion of hospital admissions (HA) for heat-related diagnoses in the total of HA was also followed. Indicators analyses are performed at local and national levels during heat waves, which are defined at the local level when forecasted biometeorological indicators have a high probability of exceeding warning thresholds [2].

\section{Results}

During both episodes, we observed a sharp increase in the number of ED attendances and GPSM visits for heat-related causes.

During the first heat wave there were a total of $3729 \mathrm{ED}$ attendances and 1456 GPSM visits for diagnoses related to heat, representing respectively $1 \%$ and $2.5 \%$ of total national activity (in comparison respectively with $0.3 \%$ and $0.6 \%$ in June 2015). A peak was recorded on the $4^{\text {th }}$ of July with 497 ED attendances and 205 GPSM visits representing up to $3 \%$ and more than $8 \%$ of total activity in the most affected regions. All age groups were concerned but the elderly were mostly represented in ED visits while children and adults less than 75 years old were mostly represented in GPSM visits. ED attendances for dehydration and hyponatremia were higher in the elderly while visits for hyperthermia/heat stroke were mostly recorded in children and adults less than 75 years old.

Heat indicators reached lower levels during the second heat wave, which affected a most restricted geographical area. About 3000 ED attendances and 610 GPSM visits (respectively $0.6 \%$ and $1.2 \%$ of total activity) were recorded.

The proportion of HA for heat-related diagnoses represented locally up to $8,2 \%$ of total HA during the first episode and up to $5,2 \%$ during the second episode in the most affected regions.

\section{Conclusions}

An increase in all heat-related indicators was observed in all age groups and particularly in the elderly during the July 2015 heatwaves. This confirms that heat-related indicators are specific and sensible.

A high proportion of HA for diagnoses related to heat in the total of $\mathrm{HA}$ is an indicator of severity and could generate local and occasional situations of tensions in the health care system, as observed during the July heat waves.

Since 2003, improvements have been made to prevent the health impacts of heat (communication, training of health professionals, access to cool rooms in nursing homes...). However, heat waves remain hazardous for population health with potential impact on ED attendances and hospitalizations.

\section{Keywords}

heat wave; impact assessment; emergency departements; healthrelated indicators

\section{Acknowledgments}

To OSCOUR $®$ emergency departments and SOS Médecins associations

\section{References}

1. Caserio-Schönemann C, Bousquet V, Fouillet A, Henry V. Le système de surveillance syndromique SurSaUD (R). Bull Epidémiol Hebd 2014;3-4:38-44.

2. Laaidi K, Ung A, Wagner V, Beaudeau P, Pascal M. The French Heat and Health Watch Warning System: principles, fundamentals and assessment. Saint-Maurice: Institut de veille sanitaire; 2013. 17 p.

*Céline Caserio-Schönemann

E-mail: c.caserio-schonemann@invs.sante.fr 